\title{
Lipoprotein Lipase Expression Exclusively in Liver A Mouse Model for Metabolism in the Neonatal Period and During Cachexia
}

Martin Merkel, ${ }^{\star}$ Peter H. Weinstock, ${ }^{\star}$ Tova Chajek-Shaul,‡ Herbert Radner,§ Baoyun Yin, *Laboratory of Biochemical Genetics and Metabolism, The Rockefeller University, New York 10021; Division of Medicine, Hadassah University Hospital, Jerusalem, Israel 91120; ${ }^{\S}$ Institute of Pathology, Karl-Franzens University, 8010 Graz, Austria; and $\|^{D e p a r t m e n t}$ of Medicine, Columbia University College of Physicians and Surgeons, New York 10032

\begin{abstract}
Lipoprotein lipase (LPL), the rate-limiting enzyme in triglyceride hydrolysis, is normally not expressed in the liver of adult humans and animals. However, liver LPL is found in the perinatal period, and in adults it can be induced by cytokines. To study the metabolic consequences of liver LPL expression, transgenic mice producing human LPL specifically in the liver were generated and crossed onto the LPL knockout (LPL0) background. LPL expression exclusively in liver rescued LPL0 mice from neonatal death. The mice developed a severe cachexia during high fat suckling, but caught up in weight after switching to a chow diet. At $18 \mathrm{~h}$ of age, compared with LPL0 mice, liver-only LPL-expressing mice had equally elevated triglycerides (10,700 vs. $14,800 \mathrm{mg} / \mathrm{dl}, P=\mathrm{NS})$, increased plasma ketones (4.3 vs. $1.7 \mathrm{mg} / \mathrm{dl}, P<0.05)$ and glucose ( 28 vs. $15 \mathrm{mg} / \mathrm{dl}, P<0.05)$, and excessive amounts of intracellular liver lipid droplets. Adult mice expressing LPL exclusively in liver had slower VLDL turnover than wild-type mice, but greater VLDL mass clearance, increased VLDL triglyceride production, and three- to fourfold more plasma ketones. In summary, it appears that liver LPL shunts circulating triglycerides to the liver, which results in a futile cycle of enhanced VLDL production and increased ketone production, and subsequently spares glucose. This may be important to sustain brain and muscle function at times of metabolic stress with limited glucose availability. (J. Clin. Invest. 1998. 102:893901.) Key words: triglycerides • fatty acids • ketone bodies • energy metabolism • glucose
\end{abstract}

\section{Introduction}

Lipoprotein lipase (LPL) ${ }^{1}$ is the rate-limiting enzyme for hydrolysis of lipoprotein triglyceride. Through hydrolysis of tri-

Address correspondence to Dr. J.L. Breslow, Laboratory of Biochemical Genetics and Metabolism, The Rockefeller University, 1230 York Avenue, Box 179, New York, NY 10021. Phone: 212-327-7700; FAX: 212-327-7165; E-mail: breslow@rockvax.rockefeller.edu

Received for publication 26 January 1998 and accepted in revised form 8 July 1998.

1. Abbreviations used in this paper: A-I-LPL, apo A-I promoter-LPL minigene; FCR, fractional catabolic rate; hLPL, human LPL; LPL, lipoprotein lipase; LPL0, LPL knockout; LPL1, heterozygote LPL knockout; LPL2, wild-type mLPL genotype; mLPL, mouse LPL; PHP, post-heparin plasma.

J. Clin. Invest.

(C) The American Society for Clinical Investigation, Inc. 0021-9738/98/09/0893/09 \$2.00

Volume 102, Number 5, September 1998, 893-901

http://www.jci.org glycerides in chylomicrons and large VLDL, LPL controls the uptake of fatty acids into tissues. In addition, based on in vitro studies, it has been postulated that LPL has nonenzymatic functions, including bridging between LDL or VLDL and cell surface and matrix proteoglycans, and is a ligand for the LDL receptor-related protein family (for reviews see references 1 and 2).

In adult animals, LPL is made in many tissues in the body, with adipose tissue and muscle accounting for most LPL production. LPL is normally not made in adult liver; however, LPL is expressed in the liver of newborn animals. Liver LPL activity rapidly declines during the first few weeks of life, while the initially low LPL in most peripheral tissues rises to adult levels (3-6). The physiological reason for LPL expression in the liver during the perinatal period is unknown.

As a response to infection or cancer, dramatic changes in lipid and energy metabolism are seen. Most pronounced are hypertriglyceridemia in combination with increased VLDL production and increased adipose tissue lipolysis, and weight loss (cachexia). In these situations, adipose tissue LPL decreases as a result of cytokine action (for reviews see references 7 and 8), while liver LPL expression can be induced in adult animals by cytokines. After a single dose of TNF, LPL mRNA and activity was found in livers of several rodent species (9-11). In addition, a markedly increased liver LPL activity has been shown in mice after tumor implantation (12). However, the importance of hepatic and peripheral LPL for developing this metabolic phenotype is not fully understood.

To explore the metabolic role of liver LPL, we created transgenic mice expressing a human LPL (hLPL) minigene driven by a liver-specific expression element in the apo A-I gene promoter. This transgene bred onto the LPL knockout (LPL0) background rescued the neonatal lethal phenotype of the LPLdeficient mice. Compared with wild-type mice, mice expressing LPL only in liver had increased liver lipid droplets, VLDL production, and plasma ketone body levels. Therefore, we postulate that liver LPL expression at times of metabolic stress, such as the perinatal period and during cachexia, shunts circulating triglyceride to the liver to provide more energy for liverspecific functions such as VLDL and ketone body production.

\section{Methods}

Construction of apo A-I-LPL minigene and generation of transgenic mice. A fragment of the human A-I gene promoter, previously shown to control liver-specific expression (13), was cloned upstream of an hLPL minigene (14). This apo A-I promoter-LPL minigene construct (A-I-LPL) was excised from the plasmid and microinjected as described (13).

Breeding of mice expressing LPL only in liver. Founder animals were crossed with wild-type $(\mathrm{CBA} / \mathrm{J} \times \mathrm{C} 57 \mathrm{Bl} / 6 \mathrm{~L}) \mathrm{F}_{1}$ mice (The Jackson Laboratory, Bar Harbor, ME), and then with heterozygote LPL knockout mice (LPL1, 15). Pups heterozygous for both LPL defi- 
ciency and the A-I-LPL transgene (LPL1/A-I-LPL) were crossed with LPL1 mice. The following genotypes resulted from this cross: $1 / 8$ wild-type (LPL2), 1/8 wild-type plus liver LPL expression (LPL2/A-ILPL), 1/4 LPL1, 1/4 LPL1/A-I-LPL, 1/8 LPL0, 1/8 LPL knockout plus liver LPL (LPL0/A-I-LPL). Littermate controls were used for all experiments.

Genotyping of induced mutant mice. Genotypes were determined from tail tip DNA by double PCR analysis as reported recently. To determine the genotype at the LPL locus, a 3-primer PCR was used. The A-I-LPL transgene was detected using a PCR for the hLPL minigene (16).

RNA analysis for tissue-specific expression of the transgene. Total cellular RNA was extracted from frozen tissues (17) and reverse transcribed into cDNA using a Gene Amp RNA PCR kit (PerkinElmer, Norwalk, CT). RT-PCR on total RNA was performed with two different specific upstream primers (5'-CCTCAAGGGAAAGCTGCCCAC-3' for hLPL and 5'-CCGAGGAATTCTGCGCCCTGTAAC- $3^{\prime}$ for mouse LPL [mLPL]) together with the same downstream primer (5'-GTTACCGTCCAGCCATGGATCACCA-3') resulting in 415 and 421 bp PCR products, respectively (18). The conditions for both reactions were 30 cycles of $93^{\circ} \mathrm{C} / 1 \mathrm{~min}, 65^{\circ} \mathrm{C} / 1 \mathrm{~min}$, and $72^{\circ} \mathrm{C} / 2 \mathrm{~min}$. Reaction products were resolved by gel electrophoresis with ethidium bromide staining.

Plasma LPL activity. LPL activity was determined in each mouse line using post-heparin plasma (PHP) from age-matched male mice $(8-10 \mathrm{wk}$ old). Fasting mice received a tail vein injection of $100 \mathrm{U} / \mathrm{kg}$ heparin (Elkins-Sinns, Cherry Hill, NJ). Blood was obtained by retroorbital bleeding $5 \mathrm{~min}$ later and plasma was frozen at $-70^{\circ} \mathrm{C}$. PHP $(10$ $\mu l)$ was assayed using a glycerol-based assay with human serum as the source of apo CII (19). This assay measured both LPL and hepatic lipase-mediated lipolysis of triglyceride. An mAb for hLPL (20) and a chicken polyclonal anti-LPL antiserum that inhibited mLPL were used alone and in combination to estimate the amount of hLPL and mLPL. Activity expressed as micromoles of FFA per hour per milliliter of PHP was determined by comparison with a standard source of hLPL of known activity. PHP from previously described mice expressing hLPL in the muscle (14) was used as a control.

Growth curves and body mass composition. Littermates from five LPL1 $\times$ LPL1/A-I-LPL(L) matings were weighed daily in the morning until weaning at age $21 \mathrm{~d}$ and then weekly on chow diet. Body mass composition was analyzed by dehydration and lipid extraction as described (21).

Histological analysis. 18-h-old pups were killed by barbiturate injection. A piece of liver was removed, and both the whole carcass and the piece of liver were fixed in neutral phosphate-buffered $10 \%$ formaldehyde solution. For lipid staining, the right half of the formalinfixed carcasses and 4- $\mu$ m-thick cryocut liver sections were stained with oil red-O and hematoxylin and eosin. The remaining half carcass and liver were embedded in paraffin wax by conventional techniques. 5 - $\mu$ m-thick paraffin sections were stained with hematoxylin and eosin. An additional liver sample was post-fixed in $3 \%$ cacodylatebuffered glutaraldehyde ( $\mathrm{pH}$ 7.3) for $4 \mathrm{~h}$, post-fixed with $1 \% \mathrm{OsO}_{4}$ in sodium cacodylate, dehydrated, and embedded in Agar 100 for azurmethylene (L)-blue stained semi-thin sections.

Lipid determination in liver tissue. After anesthesia, blood was removed from the left ventricle, and mice were perfused with $0.9 \%$ $\mathrm{NaCl}$ solution. Livers were excised, weighed, and frozen. Total lipids were extracted from organs (22). Liver triglycerides were quantified using TLC and ester determination (23). Total and free cholesterol were measured using a Perkin-Elmer gas-liquid chromatograph with coprostinol as a standard as described (24).

Lipid, lipoprotein, ketone body, and glucose analysis. Mice were fed a chow diet $(4.5 \% \mathrm{wt} / \mathrm{wt}$ fat). Blood was collected from 18 -h-old pups by cardiac puncture. From adult mice, blood samples were taken by retroorbital puncture after $8 \mathrm{~h}$ of daytime fasting. Plasma triglyceride, cholesterol, ketone bodies, and glucose were determined using commercial kits, which were adapted for 96-well microtiter plates (Sigma Chemical Co., St. Louis, MO; \#334, \#352, \#310, and
\#315, respectively). Lipoprotein separation was performed by gel filtration chromatography of $200 \mu \mathrm{l}$ pooled plasma on two serial Superose 6 columns (FPLC; Pharmacia, Uppsala, Sweden). Fractions of 0.3 $\mathrm{ml}$ were analyzed for cholesterol and triglyceride. In addition, plasma samples from individual mice were analyzed by sequential ultracentrifugation (25).

$V L D L$ turnover study. In vivo labeling of VLDL with radioactive triglycerides was performed using $500 \mu \mathrm{Ci}\left[1-{ }^{14} \mathrm{C}\right]$ palmitic acid $(\mathrm{Am}$ ersham, Arlington Heights, IL) as described (26). In addition, VLDL was labeled with $\left[1,2(n)-{ }^{3} \mathrm{H}\right]$ cholesteryl oleyl ether (Amersham) as follows: VLDL from 10 mice was isolated by ultracentrifugation, and $5 \mu \mathrm{g}$ purified CETP (provided by Dr. A. Tall, Columbia University) was added. The mixture was added to $500 \mu \mathrm{Ci}$ dried $\left[{ }^{3} \mathrm{H}\right]$ cholesteryl oleyl ether and rocked overnight at $37^{\circ} \mathrm{C}$. Labeled VLDL was isolated with a Sephadex G-50 column (Pharmacia). Anesthetized male mice were injected with $10^{5} \mathrm{dpm}\left[{ }^{14} \mathrm{C}\right]$ triglyceride and $10^{6} \mathrm{dpm}$ $\left[{ }^{3} \mathrm{H}\right]$ cholesterol oleyl ether-labeled VLDL into their femoral vein. The rate of disappearance of labeled VLDL was determined by measuring the remaining radioactivity in $10 \mu l$ total plasma drawn $2,5,10$, $20,30,45$, and 60 min after injection. Data were analyzed for individual animals using a two-pool model, and the fractional catabolic rate (FCR) was calculated.

$V L D L$ production study. Anesthetized male mice were injected intravenously with $500 \mathrm{mg} / \mathrm{kg}$ of Triton WR 1339 (Sigma) using a $15 \%$ (wt/vol) solution. After $15 \mathrm{~min}, 100 \mu \mathrm{Ci}\left[2-{ }^{3} \mathrm{H}\right]$ glycerol (Amersham) was injected intravenously. Animals were bled from the retroorbital plexus $15,30,60$, and 90 min after $\left[{ }^{3} \mathrm{H}\right]$ glycerol injection. Total lipids were extracted from serum samples and the radioactivity was measured (26). The triglyceride production rate was calculated as the increase of radioactivity in the lipid extract between 15 and $30 \mathrm{~min}$. To calculate the absolute triglyceride production, plasma triglyceride levels were measured before and $45 \mathrm{~min}$ after Triton injection.

Statistical analysis. Results are given as mean \pm SD. Statistical significance was tested using two-tailed Student's $t$ test if not otherwise stated. In certain cases, ANOVA with a Newman-Keuls post-test for multiple comparisons was used. Statistical analysis was made using the computer program Prism (GraphPad Software, San Diego, CA).

\section{Results}

Generation of transgenic mice. Transgenic mice were generated with an hLPL-minigene driven by a liver-specific fragment of the apo A-I gene promoter. Two of eight transgenic mice born after microinjection were used to develop lines. Transgene-positive males of both lines were used for further breeding with wild-type and LPL1 mice.

To verify transgene expression, PHP was obtained from wild-type mice and both A-I-LPL transgenic lines, and analyzed for hLPL, mLPL, and hepatic lipase activities. As shown in Table I, wild-type mice had an insignificant amount of hLPL activity, whereas the two A-I-LPL transgenic lines had $1.7 \pm 0.9$ and 3.8 $\pm 1.7 \mu \mathrm{mol}$ released $\mathrm{FFA} / \mathrm{ml} / \mathrm{h}$ of hLPL activity, respectively. No significant differences were found between the wildtype mice and any of the transgenic lines in mLPL or hepatic lipase activity. The A-I-LPL transgenic line with lower PHP hLPL activity was designated low (L), and the other high $(\mathrm{H})$ expressor.

To verify the tissue-specific pattern of transgene expression, tissues from both lines of A-I-LPL mice were examined by RT-PCR analysis for the presence of hLPL and mLPL mRNA. As shown in Fig. 1, both A-I-LPL transgenic lines expressed hLPL mRNA only in liver, with the signal of the L line slightly weaker than the $\mathrm{H}$ line. hLPL mRNA was absent from all other organs examined. As expected, mLPL mRNA was absent from liver but present in heart, skeletal muscle, adipose 
Table I. hLPL, mLPL, and Hepatic Lipase (HL) Activities in PHP ( $\mu \mathrm{mol}$ Released FFA per $\mathrm{ml}$ and $h$ ) from Wild-Type

(LPL2), Low and High Liver LPL-expressing Lines (LPL2/A-I-LPL[L] and LPL2/A-I-LPL[H])

\begin{tabular}{lcccc}
\hline \multicolumn{1}{c}{ Genotype } & $n$ & $\mathrm{hLPL}$ & $\mathrm{mLPL}$ & $\mathrm{HL}$ \\
\hline & & $\mu \mathrm{mol} / \mathrm{ml} / \mathrm{h}$ & $\mu \mathrm{mol} / \mathrm{ml} / \mathrm{h}$ & $\mu \mathrm{mol} / \mathrm{ml} / \mathrm{h}$ \\
& 4 & $0.42 \pm 0.39$ & $6.30 \pm 1.26$ & $11.77 \pm 1.38$ \\
LPL2 & 4 & $1.66 \pm 0.89$ & $8.70 \pm 1.10$ & $11.60 \pm 1.54$ \\
LPL2/A-I-LPL(L) & 7 & $3.80 \pm 1.65$ & $8.21 \pm 3.18$ & $11.02 \pm 2.50$ \\
LPL2/A-I-LPL(H) & 7 & $<0.005^{*}$ & $\mathrm{NS}$ & $\mathrm{NS}$ \\
P ANOVA & & & & \\
& & & &
\end{tabular}

*Newman-Keuls post-test: $P=$ NS for LPL2 vs. LPL2/A-I-LPL(L); $P<0.01$ for LPL2 vs. LPL2/A-I-LPL(H) $P<0.05$ for LPL2/A-ILPL(L) vs. LPL2/A-I-LPL(H).

tissue, and kidney. These studies established that the A-I-LPL transgenic mice express hLPL exclusively in liver.

Breeding and survival. The low and the high liver LPLexpressing lines were bred with LPL1 mice and the LPL1/A-ILPL offspring were again crossed with LPL1 mice. The expected numbers of mice with the different genotypes (LPL2, LPL2/A-I-LPL, LPL1, LPL1/A-I-LPL, LPL0, and LPL0/A-ILPL) were found in newborn pups (data not shown). As anticipated, all LPL0 mice died within $24 \mathrm{~h}$ (15). Survival of the LPL0/A-I-LPL mice varied depending on the transgenic line and the litter size. From 11 matings of LPL1 females with LPL1/A-I-LPL(L) males, 92 pups were born containing 11 LPL0/A-I-LPL(L) mice. Out of these, one LPL0/A-I-LPL(L) died within 24 h, six died between days 5 and 12 of age. Four mice $(36 \%)$ lived longer than 4 mo. The survival in this line was greatly improved by combining litters, i.e., placing two or more litters of the same age in one cage and removing one mother. In the monitored litters, 14 out of 17 LPL0/A-ILPL(L) mice ( $>80 \%$ ) survived the suckling period. In the high expressing line, all LPL0/A-I-LPL(H) pups died between days 5 and 14 of life (average time to death was $8.7 \pm 4.2 \mathrm{~d}$ ). Therefore, the A-I-LPL transgene prevented the neonatal death of LPL0 mice in both lines. Some LPL0/A-I-LPL mice died within the second week of life. This included all LPLO/A-

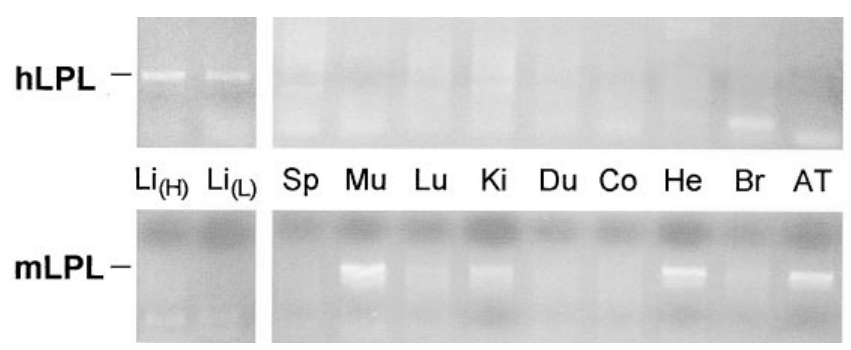

Figure 1. Organ distribution of hLPL and mLPL expression. RTPCR with total RNA from various organs was performed using primers specific for hLPL and mLPL mRNA. In the livers of the high $(\mathrm{H})$ and low (L) A-I-LPL-expressing lines only hLPL mRNA was found. The intensity of these bands corresponded to the differences in PHP hLPL activities. No other examined organ contained hLPL mRNA. mLPL mRNA showed the expected tissue distribution. Liver $(\mathrm{Li})$, spleen $(S p)$, skeletal muscle $(M u)$, lung $(L u)$, kidney $(K i)$, duodenum $(\mathrm{Du})$, colon $(\mathrm{Co})$, heart $(\mathrm{He})$, brain $(\mathrm{Br})$, adipose tissue $(A T)$.
I-LPL(H) mice and most of the LPL0/A-I-LPL(L) mice from small litter sizes. Low liver LPL expression and big litters were able to significantly increase survival.

Growth curves and body composition. The body weight of all genotypes was observed during suckling and after weaning onto chow diet. No significant differences were observed between LPL2, LPL2/A-I-LPL(L), LPL1, and LPL1/A-ILPL(L). LPL2 and LPL0/A-I-LPL(L) looked the same at birth, but by $3 \mathrm{~d}$ the LPL0/A-I-LPL(L) had failed to gain as much weight as the wild-type (Fig. 2, LPL2: 2.4 $\pm 0.14 \mathrm{~g}$; LPL0/ A-I-LPL(L): $2.1 \pm 0.12 \mathrm{~g}, P<0.05)$. The differences were most evident just before weaning (21 d: LPL2: 12.2 $\pm 1.02 \mathrm{~g}$, LPL0/ A-I-LPL(L): $8.7 \pm 0.9 \mathrm{~g}, P<0.005)$. At this time, body mass composition analysis revealed a lower percentage of fat in the LPL0/A-I-LPL(L) $\quad(5.6 \pm 0.2 \%$ fat $)$ compared with control LPL2 $(6.5 \pm 0.6 \%, P<0.05)$, whereas body water and lean body mass were not different. On a chow diet $(4.5 \%$ calories from fat), LPL0/A-I-LPL(L) mice caught up in weight. By 2-3 mo of age, the LPL0/A-I-LPL(L) and the wild-type mice were

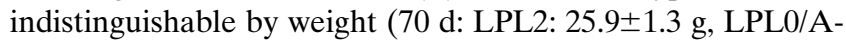
I-LPL(L): $24.4 \pm 2.3 \mathrm{~g}, P=\mathrm{NS}$ ). Therefore, the high carbohydrate chow diet corrected the growth retardation seen during intake of the high-fat suckling diet.

Liver histology and lipid content. Pathologic examination was performed at $18 \mathrm{~h}$ of age in mice of various genotypes. LPL2, LPL2/A-I-LPL(L), LPL0, and LPL0/A-I-LPL(L) mice did not differ in gross pathology. LPL2 and LPL2/A-I-LPL(L) mice had normal liver architecture with some intracellular lipid vacuoles (hematoxylin and eosin: Fig. 3, $A$ and $B$; oil red-O: Fig. 3, $I$ and $J$ ). LPL0 mice had normal liver architecture, but intracellular lipid vacuoles were very small or absent with mainly extracellular lipid (Fig. 3, $C$ and $K$ ). LPL0/A-I-LPL(L) mice also had normal liver architecture, but hepatocytes were

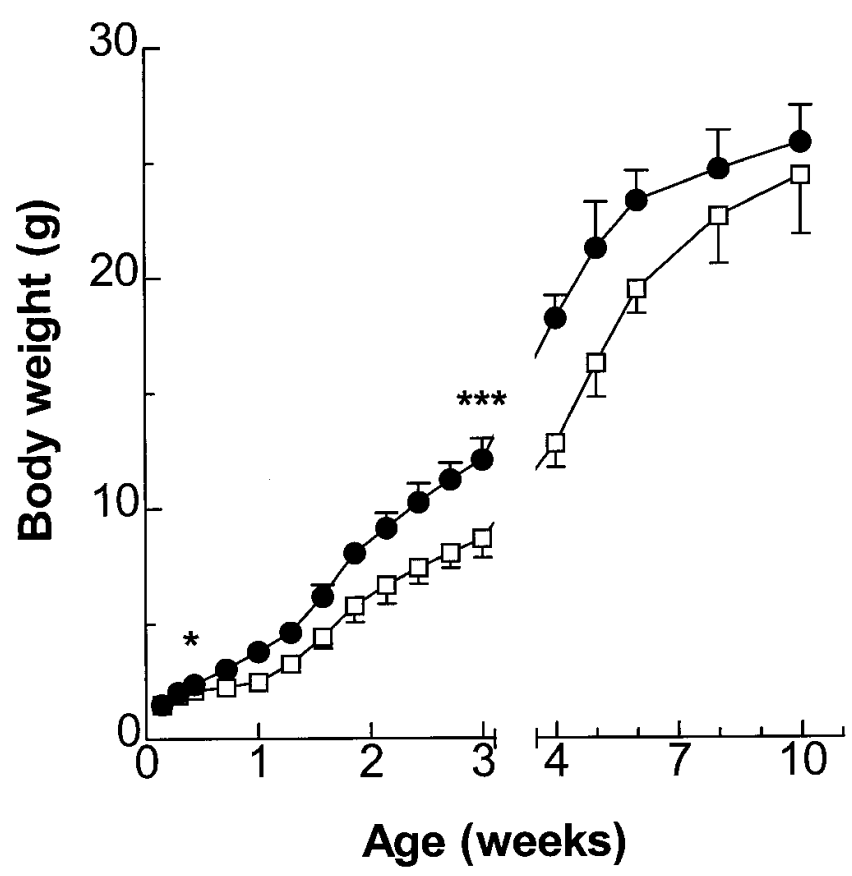

Figure 2. Growth curve of mice expressing LPL exclusively in the liver. Five mice per genotype were weighed in the morning daily before and weekly after weaning at day 21. LPL2 (filled circles), LPL0/ A-I-LPL(L) (open squares). $* P<0.05$ at $3 \mathrm{~d} ; * * * P<0.005$ at $21 \mathrm{~d}$. 
LPL2

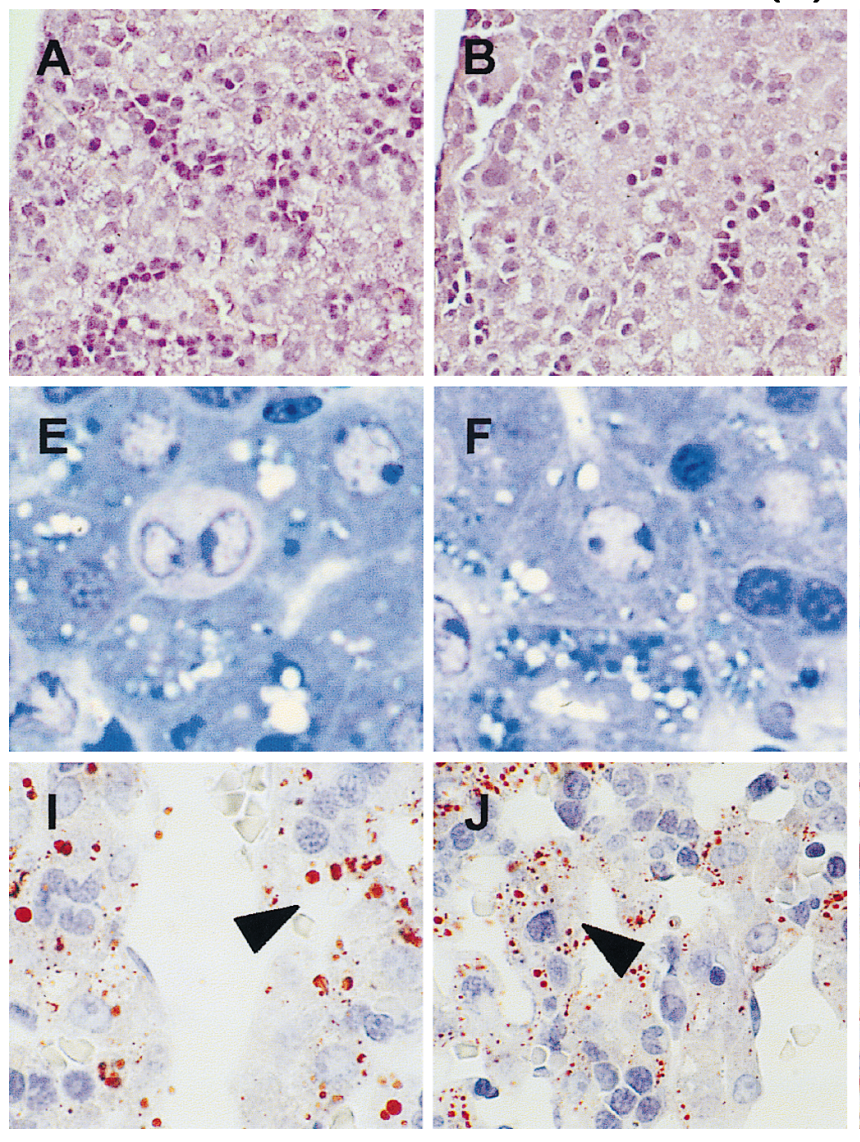

LPL2/A-I-LPL(L)
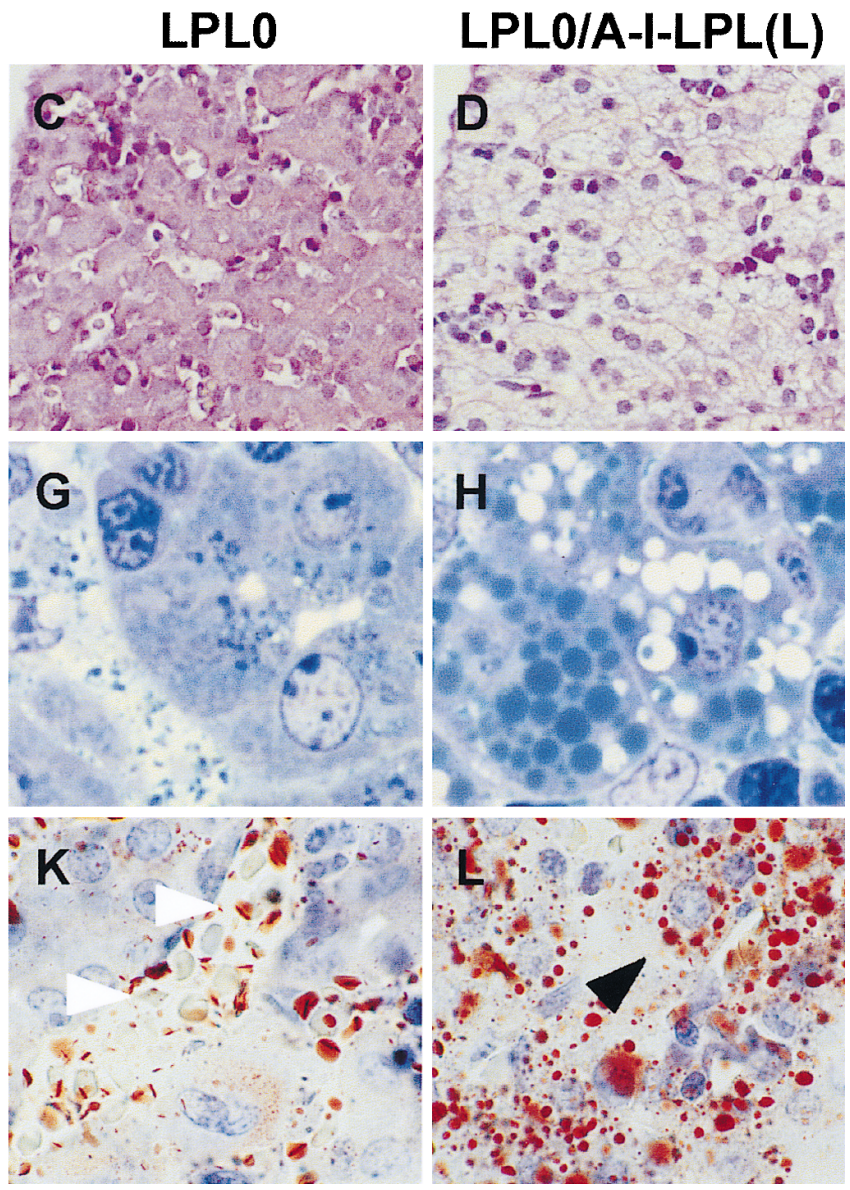

Figure 3. Liver histology of 18-h-old mice. Liver-only LPL expressors (LPL0/A-I-LPL[L]) had massive intracellular lipid storage in the liver at $18 \mathrm{~h}$ of life $(D, H$, and $L)$, whereas LPL knockout mice had no intracellular lipids in the liver $(C, G$, and $K)$. There was no obvious difference between LPL2 $(A, E$, and $I)$ and LPL2/A-I-LPL(L) $(B, F$, and $J)$. $(A-D)$ Routine staining (hematoxylin and eosin, 56× enlarged); $(E-H)$ semi-thin sections (azur-methylene-blue, $280 \times$ ). (I-L) Lipids (oil red-O, 280×). (Black arrows) Intracellular lipid droplets; (white arrows) extracellular, intravascular lipids.

filled with lipid vacuoles (Fig. 3, $D$ and $L$ ). These lipid vacuoles are also seen as the green and white circular structures in the azur-methylene-blue semi-thin sections (Fig. 3, E-H). These data suggested that liver LPL expression does not dramatically alter liver lipid storage on the LPL2 background, but causes a massive liver lipid influx and storage in LPL0/A-ILPL(L) mice, which is completely absent in LPL0 animals.

At $7 \mathrm{~d}$ of age, the livers of LPL0/A-I-LPL(L) mice were yellow and slightly bigger than those of control mice. Microscopic examination showed LPL0/A-I-LPL(L) hepatocytes around the central vein completely filled with vacuoles, with some intact cells remaining in the periphery of the liver lobule. There were no gross or histological differences between livers from LPL2, LPL2/A-I-LPL(L), LPL1, and LPL1/A-I-LPL(L) mice. At the same age, liver triglycerides were not markedly different between LPL2 and LPL2/A-I-LPL(L) mice, but were greatly increased in LPL0/A-I-LPL(L) mice (average LPL2: $16.8[n=2]$ vs. LPL0/A-I-LPL(L): $117[n=2] \mu \mathrm{mol} / \mathrm{g}$ wet liver). These differences were less pronounced in adult animals (average LPL2: 11.5 [ $n=2]$ vs. LPL0/A-I-LPL(L): $21.5[n=2]$ $\mu \mathrm{mol} / \mathrm{g}$ wet liver); LPL2/A-I-LPL(L) livers were again not different from LPL2. Interestingly, free cholesterol and cholesterol ester were not increased in livers of LPL0/A-I-LPL(L) mice (data not shown). Therefore, the liver triglyceride increase was greater in young LPL0/A-I-LPL(L) mice on the high fat suckling diet than in chow fed adult LPL0/A-I-LPL(L) mice.

Plasma lipids and lipoproteins. As shown in Table II, compared with adult wild-type mice (LPL2), transgenic mice expressing hLPL in the liver on the LPL2 background had reduced triglycerides with an 8 and $19 \%$ decrease in $\mathrm{L}$ and $\mathrm{H}$ expressor mice, respectively. Compared with adult heterozygous LPL knockout mice (LPL1), transgenic mice expressing hLPL in the liver on the LPL1 background had an even greater triglyceride reduction, $11 \%$ for $\mathrm{L}$ and $28 \%$ for $\mathrm{H}$ lines. Adult homozygous LPL knockout mice rescued by the hLPL low expressor transgene still had markedly elevated triglyceride levels $(3,310 \pm 999 \mathrm{mg} / \mathrm{dl})$. Gel filtration chromatography of lipoproteins revealed that the transgene on the LPL2 background lowered VLDL triglyceride, and on the LPL0 background resulted in a lipoprotein pattern characterized by extremely elevated VLDL triglycerides and cholesterol, and reduced HDL cholesterol.

Plasma lipids were also measured at $18 \mathrm{~h}$ of age on the high fat suckling diet. As shown in Table III, the hLPL low expressor transgene lowered triglyceride levels on the LPL2, LPL1, 
Table II. Triglyceride and Cholesterol Levels of Adult Mice of the Low $(L)$ and High $(H)$ Liver LPL-expressing Lines on the Wild-Type (LPL2), Heterozygote LPL Knockout (LPL1) and LPL Knockout (LPLO) Background

\begin{tabular}{lccc}
\hline & $n$ & Triglycerides & Cholesterol \\
\hline & & $m g / d l$ & $m g / d l$ \\
& & & \\
LPL2 background & 22 & $86.3 \pm 6.86$ & $92.6 \pm 11.1$ \\
LPL2* & 10 & $79.0 \pm 13.2$ & $87.6 \pm 17.9$ \\
LPL2/A-I-LPL(L) & 11 & $69.6 \pm 13.1$ & $89.1 \pm 17.2$ \\
LPL2/A-I-LPL(H) & & $<0.001^{\ddagger}$ & $\mathrm{NS}$ \\
$P$ ANOVA & & & \\
LPL1 background & 24 & $142 \pm 21.1$ & $78.6 \pm 15.5$ \\
LPL1* & 12 & $127 \pm 14.8$ & $67.5 \pm 17.7$ \\
LPL1/A-I-LPL(L) & 13 & $102 \pm 26.3$ & $70.5 \pm 16.0$ \\
LPL1/A-I-LPL(H) & & $<0.0001^{\S}$ & $\mathrm{NS}$ \\
$P$ ANOVA & 6 & $3310 \pm 999.4$ & $287 \pm 79.0$ \\
LPL0/A-I-LPL (L) & & $<0.0001$ & $<0.0001$ \\
$P$ LPL2 vs. LPL0/A-I-LPL(L), $t$ test & & &
\end{tabular}

${ }^{\ddagger}$ Newman-Keuls post-test: $P=$ NS for LPL2 vs. LPL2/A-I-LPL(L); $P<$ 0.001 for LPL2 vs. LPL2/A-I-LPL(H); $P<0.05$ for LPL2/A-I-LPL(L) vs. LPL2/A-I-LPL(H); ${ }^{\circledR}$ Newman-Keuls post-test: $P<0.05$ for LPL1 vs. LPL1/A-I-LPL(L); $P<0.001$ for LPL1 vs. LPL1/A-I-LPL(H); $P<0.01$ for LPL2/A-I-LPL(L) vs. LPL2/A-I-LPL(H). *Littermate controls were used. Controls from low and high expressing lines were not significantly different and the groups were pooled.

and LPL0 backgrounds. On the LPL2 and LPL1 backgrounds the percent decrease in triglycerides caused by the transgene at $18 \mathrm{~h}$ of age was even greater than in adult mice. At $18 \mathrm{~h}$ of age on the LPL0 background, the transgene lowered triglyceride levels, but these still remained markedly elevated.

VLDL turnover and production. To define how liver LPL expression altered VLDL metabolism in the mice, several metabolic studies were done. Double-labeling of VLDL with $\left[{ }^{14} \mathrm{C}\right]$ palmitic acid and $\left[{ }^{3} \mathrm{H}\right]$ cholesteryl oleyl ether enabled us to observe the hydrolysis of VLDL triglycerides as well as the fate of nonhydrolyzable core lipids that served as a marker for VLDL particle turnover. The results shown in Fig. 4, $A$ and $B$, include computer-generated curves using a two-pool exponential decay model. For the FCR calculation, the 2-60-min

Table III. Triglyceride Levels of 18-h-old Pups

\begin{tabular}{lcc}
\hline \multicolumn{1}{c}{ Genotype } & $n$ & Triglycerides \\
\hline & & $m g / d l$ \\
LPL2 & 13 & $126.2 \pm 29.6$ \\
LPL2/A-I-LPL(L) & 10 & $97.0 \pm 22.9$ \\
$\quad P$ LPL2 vs. LPL2/A-I-LPL(L), $t$ test & & $<0.05$ \\
LPL1 & 17 & $398 \pm 179$ \\
LPL1/A-I-LPL(L) & 20 & $217 \pm 109$ \\
$P$ LPL1 vs. LPL1/A-I-LPL(L), $t$ test & & $<0.001$ \\
LPL0 & 9 & $14788 \pm 4199$ \\
LPL0/A-I-LPL(L) & 8 & $10675 \pm 4273$ \\
$P$ LPL0 vs. LPL0/A-I-LPL(L), $t$ test & & $\mathrm{NS}$ \\
& & \\
\hline
\end{tabular}

plasma radioactivity data were used except for the triglyceride decay in LPL2 and LPL2/A-I-LPL(L) animals. For the latter, only 2-30-min data were used since thereafter $>90 \%$ of the injected label had been cleared.

On the wild-type background, VLDL triglyceride hydrolysis was significantly faster than whole particle uptake as shown by VLDL cholesteryl ether. Liver LPL tended to increase the FCR for both labels, but these differences were not significant (VLDL triglyceride: $7.6 \pm 3.7$ and $9.5 \pm 5.1$ pools/h; VLDL cholesterol ether: $0.74 \pm 0.02$ and $0.96 \pm 0.13$ pools $/ \mathrm{h}$ ). The total mass clearance from plasma (FCR multiplied by the pool size, Fig. 4, $C$ and $D$ ) for VLDL triglycerides was 7.0 3 3.7 (LPL2) and $7.3 \pm 2.8 \mathrm{mg} / \mathrm{h}$ (LPL2/A-I-LPL[L]) and for VLDL cholesterol esters $0.069 \pm 0.025$ and $0.077 \pm 0.04 \mathrm{mg} / \mathrm{h}$. LPL0/A-ILPL(L) animals had a significantly slower FCR for both substrates compared with LPL2 mice (VLDL triglycerides: $0.36 \pm 0.04$ pools $/ \mathrm{h}, P<0.01$; VLDL cholesteryl ether $0.51 \pm 0.05$ pools/h, $P<0.001)$. However, due to the high plasma lipid levels in LPL0/A-I-LPL(L), the mass clearance from plasma was markedly increased (VLDL triglycerides: $14.0 \pm 2.4 \mathrm{mg} / \mathrm{h}, P<$ 0.05 , Fig. $4 C$; VLDL cholesterol esters $1.2 \pm 0.1 \mathrm{mg} / \mathrm{h}, P<$ 0.0001, Fig. $4 \mathrm{D}$ ). Unlike LPL2 animals, LPL0/A-I-LPL(L) showed a faster turnover for VLDL cholesteryl ether than for VLDL triglycerides.

These VLDL mass clearance data suggested that VLDL production rates were increased in LPL0/A-I-LPL(L) mice. To confirm this, VLDL production rates were estimated using triglyceride determination before and after Triton injection as well as in vivo VLDL-triglyceride labeling with $\left[{ }^{3} \mathrm{H}\right]$ glycerol. As shown in Fig. 5 A, the triglyceride increase 45 min after Triton injection was significantly greater in LPL0/A-I-LPL(L)

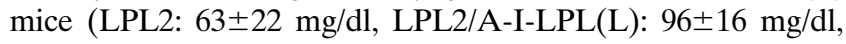
$P=$ NS, LPL0/A-I-LPL(L): $1,557 \pm 230 \mathrm{mg} / \mathrm{dl}, P<0.0001$ vs. LPL2). The $\left[{ }^{3} \mathrm{H}\right]$ glycerol labeling confirmed a greater VLDL production rate in LPL0/A-I-LPL(L) (slope 15 to $30 \mathrm{~min}$ : LPL2: 14 \pm 2.7 , LPL2/A-I-LPL(L): 17 $\pm 7.1, P=$ NS, LPL0/A-ILPL(L): $34 \pm 3.1, P<0.0005)$. Therefore, compared with LPL2 and LPL2/A-I-LPL(L) mice, the A-I-LPL transgene in LPLO/ A-I-LPL(L) mice mediated a higher mass clearance of VLDL triglyceride and whole VLDL particles into the liver. As a result of this, the VLDL production rate was dramatically increased in LPL0/A-I-LPL(L) animals.

Ketone bodies and plasma glucose. To test whether greater uptake of triglyceride by the liver would alter the plasma concentrations of other energy sources, ketones and glucose were measured. At $18 \mathrm{~h}$ of age, both LPL2/A-I-LPL(L) and LPL0/ A-I-LPL(L) had almost threefold higher ketones than LPL2 and LPL0 mice, respectively (Fig. 6 A: LPL2: 1.4 $1.1 \mathrm{mg} / \mathrm{dl}$; LPL2/A-I-LPL(L) 3.8 $\pm 1.8, P<0.05$ vs. LPL2; LPL0: 1.7 \pm 2.5 ; LPL0/A-I-LPL(L): $4.3 \pm 2.0, P<0.05$ vs. LPL0). Fasted adult LPL0/A-I-LPL(L) mice also had three- to fourfold more plasma ketone bodies (LPL2: $2.8 \pm 1.1 \mathrm{mg} / \mathrm{dl}$; LPL2/A-ILPL(L): $2.7 \pm 1.0, P=$ NS vs. LPL2; LPL0/A-I-LPL(L): 9.5 $\pm 2.6, P<0.01$ vs. LPL2). At $18 \mathrm{~h}$ of age, LPL2 and L2/A-ILPL(L) had glucose levels of $85 \pm 17$ and $82 \pm 16 \mathrm{mg} / \mathrm{dl}$, respectively. At the same age, glucose levels were only $15 \pm 8.5 \mathrm{mg} / \mathrm{dl}$ in LPL0 pups, whereas LPL0/A-I-LPL(L) pups had significantly higher glucose, $28 \pm 7.6 \mathrm{mg} / \mathrm{dl}(P<0.05$ vs. LPL0, Fig. 6 $B)$. Therefore, both adult and neonatal LPL0/A-I-LPL(L) mice had markedly elevated ketone body levels. The severe neonatal hypoglycemia found in the LPL0 mice was somewhat alleviated by the A-I-LPL transgene. 

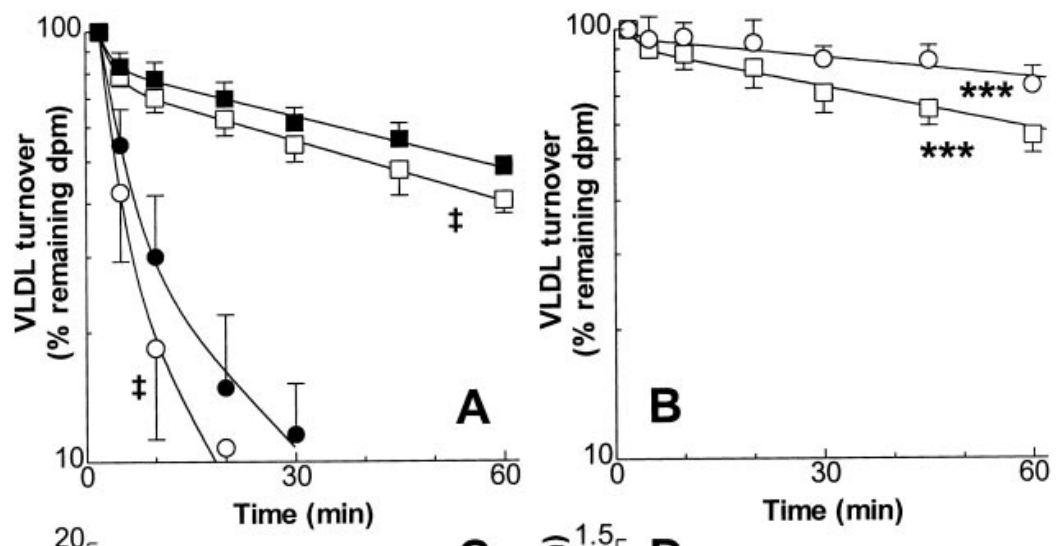

Figure 4. VLDL turnover and mass clearance study. VLDL was labeled with $\left[{ }^{14} \mathrm{C}\right]$ palmitate to observe triglyceride hydrolysis (circles) and with $\left[{ }^{3} \mathrm{H}\right]$ cholesteryl oleyl ether as a marker for whole VLDL particle turnover (squares). (A) VLDL turnover in LPL2 (filled circles and filled squares) and in LPL2/A-I-LPL(L) (open circles and open squares) mice. (B) VLDL turnover in liver-only LPL-expressing mice (LPL0/A-ILPL[L]). Computer-generated slopes are shown using a two-pool model. The 2-min radioactivity was set $100 \%$ for each curve. The FCR for both labels was significantly less in LPL0/A-I-LPL(L) compared with LPL2. (C) VLDL triglyceride mass clearance, $(D)$ VLDL cholesterol ester mass clearance. The mass uptake in LPL0/A-I-LPL(L) was markedly increased compared with LPL2 due to their high VLDL plasma pool. ${ }^{\ddagger}$ LPL2/A-I-LPL mice had a nonsignificant trend towards faster turnover and higher mass uptake compared with LPL2 for both labels. $* P<0.05 ; * * * P<$ 0.0001; each vs. LPL2 of same label.

\section{Discussion}

A mouse was created expressing LPL exclusively in the liver using an hLPL minigene driven by a fragment of the apo A-I gene promoter that directs liver-specific expression. The transgene was shown to be metabolically active by demonstrating in two transgenic lines that the degree of reduction of plasma triglycerides was proportional to the level of transgene expression, and that the transgene on the LPL knockout background was able to rescue the neonatal lethality of that genotype.

The fact that LPL0/A-I-LPL(L) mice survived without a dramatic decrease in triglyceride levels compared with neonatal LPL0 mice was somewhat unexpected. Neonatal death in LPLO mice and another mutant mouse deficient in LPL activity, the cld mouse, had been thought to be due to extremely high triglyceride levels causing pulmonary capillary obstruction and microinfarcts of other organs $(15,27,28)$. The survival of the LPL0/A-I-LPL(L) mice suggests an alternative explanation. Newborn animals require a source of energy for the central nervous system, heart, and respiratory muscles. This is supplied mainly by dietary glucose and lipid, since glycogen stores, tissue fat deposits, and muscle mass are all minimal in the newborn period. In the suckling mouse the main dietary energy source is fat, $70 \%$ of calorie intake, only $7.5 \%$ of calories are from carbohydrate (29), compared with 55\% from fat and $39 \%$ from carbohydrates in human milk (30). Mice with complete LPL deficiency cannot take up dietary fatty acids from plasma triglyceride in peripheral tissues $(15,21)$, so these animals require dietary glucose as their main energy source. Therefore, glucose scarcity may contribute to the neonatal death of LPL-deficient mice.

This hypothesis is supported by the low glucose levels found in newborn LPL0 mice. LPL0/A-I-LPL(L) mice have another source of energy which is the utilization of dietary fat by the liver for ketone body production. Ketone bodies can be used by brain and muscle and could relieve the metabolic burden imposed by relative glucose scarcity in newborn LPL-deficient mice. Newborn LPL0/A-I-LPL(L) pups have markedly higher ketone body levels than wild-type animals, whereas LPL0 mice have not. In human newborns, there is a similar increase in plasma ketones to $5-10 \mathrm{mg} / \mathrm{dl}$, which can be pre-
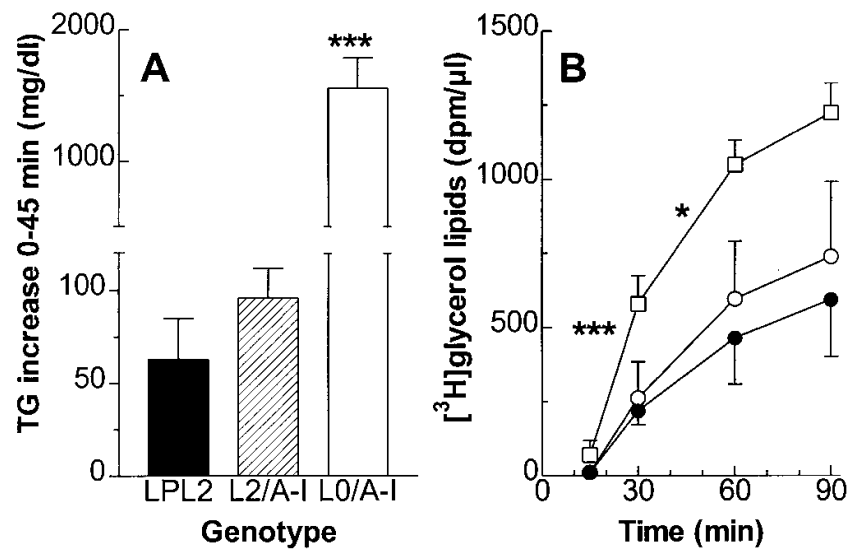

Figure 5. VLDL triglyceride production rate. $(A)$ Triglyceride concentration was determined before and $45 \mathrm{~min}$ after Triton WR 1339 injection. (B) $15 \mathrm{~min}$ after Triton injection, $\left[{ }^{3} \mathrm{H}\right]$ glycerol was injected and radioactivity in plasma lipids was determined at different time points. LPL2 (filled circles), LPL2/A-I-LPL(L) (open circles), LPL0/ A-I-LPL(L) (open squares). In both experiments, LPL0/A-I-LPL(L) showed a markedly higher VLDL production rate. $* P<0.05$; *** $P<$ 0.0005 ; both vs. LPL2. 

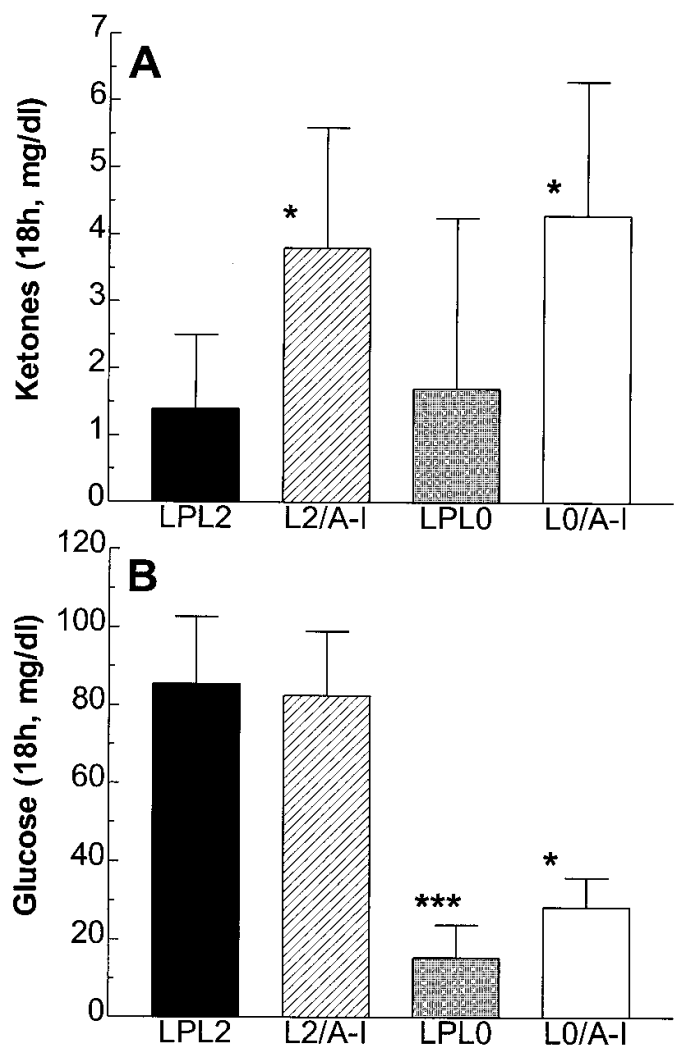

Figure 6. Plasma ketone and glucose levels in 18-h-old mice. $(A)$ Both LPL2/A-I-LPL(L) and LPL0/A-I-LPL(L) had significantly higher ketones than LPL2 and LPL0 mice. $(B)$ The severe neonatal hypoglycemia found in the LPL0 mice was somewhat alleviated by the A-I-LPL(L) transgene. $(A) * P<0.05$ vs. LPL2 and LPL0, respectively. $(B) * P<0.05$ vs. LPL0; $* * * P<0.0001$ vs. LPL2.

vented by glucose infusion or feeding (31). Therefore, in the LPL0/A-1-LPL pups it is likely that ketone body utilization in peripheral organs led to a glucose saving effect resulting in the observed significantly higher plasma glucose levels in newborn LPL0/A-I-LPL(L) compared with LPL0 mice. Thus, the lack of energy for crucial metabolic processes in brain and muscle may be another explanation for the neonatal death of LPLdeficient mice, and the formation of ketone bodies by the liver-only LPL expressors may allow their survival.

In the neonatal period the most profound pathological difference between LPLO and LPLO/A-I-LPL(L) mice was the presence of large amounts of hepatocellular lipid droplets in the liver LPL-expressing lines. In the LPL-deficient state, lipoprotein triglyceride was not taken up by the liver, and no liver lipid droplets were found. This strongly suggests that in this situation liver LPL is a gatekeeper for the entry of lipoprotein triglyceride into liver, as it is into peripheral tissues of normal animals. Thus, by routing triglyceride to the liver, the physiological LPL expression in livers of normal neonatal animals may optimize energy metabolism during this period of relative peripheral LPL deficiency and limited energy substrate availability.

Although LPL0/A-I-LPL mice survived the neonatal period, many of them died between ages 1 and 2 wk. Surprisingly, especially the animals with the greater hLPL expression, LPL0/A-I-LPL(H), did not survive to adulthood. These data and the greater survival of mice that were suckled in larger litters suggested that the demise in the second week of life was the result of a different process than the neonatal death of LPLO mice. Perhaps hepatocellular lipid accumulation was greater in the A-I-LPL(H) line and resulted in severe hepatic dysfunction.

If energy deficiency leads to neurological or respiratory dysfunction in LPL0 mice, why do humans with complete LPL deficiency survive? Since most humans with LPL deficiency have inactive rather than absent LPL protein, it is possible that the inactive forms of LPL allow some triglyceride uptake into tissue. However, there are other more likely explanations for the survival of LPL-deficient humans. First, carbohydrates provide a much greater percentage of total calories in human milk than they do in mice (40 vs. $7.5 \%$ ). Therefore, infants with LPL deficiency may ingest enough carbohydrate to provide for their basic metabolic requirements. Second, unlike in mice where hepatic lipase is predominantly found in the circulating blood, the human enzyme remains in the liver (32), where it may function as a secondary mechanism for liver lipid uptake.

Liver-only expression of LPL markedly altered the metabolism of VLDL. Metabolic studies showed that liver LPL on the knockout background led to a greatly increased mass clearance of triglyceride and nonhydrolyzable core lipid compared with wild-type mice. These lipids are taken up by the liver, as also evidenced by histologically found liver lipid droplets in these animals. Most likely as a direct response to the increased liver lipid uptake, the triglyceride production rate was greatly increased in the LPL0/A-I-LPL(L) mice. Despite this, the mouse livers may have been unable to synthesize and secrete enough lipoproteins to keep up with the remarkably increased lipid influx and they stored part of this as lipid droplets.

In LPL2 and LPL2/A-I-LPL(L) mice, triglyceride was removed much more rapidly from plasma than core cholesteryl ether. Most likely this was because peripheral LPL hydrolyzed VLDL triglycerides quickly and the newly liberated FFA were rapidly taken up by the adjacent tissues. The slower lipid removal in the LPL0/A-I-LPL(L) mice was in accord with the lower total body LPL activity, as shown by PHP activities. Surprisingly, the cholesteryl ether label disappeared from the circulation at a faster rate than did the triglyceride. Our expectation was that in these animals both labels would be removed at an equal rate or with a faster removal of hydrolyzable triglyceride, depending on whether LPL-mediated whole particle uptake or hydrolysis was the predominant mechanism. One reason for this may be that some radioactive surface lipids could have been shed during hydrolysis and not taken up by the liver. Alternatively, labeled fatty acids from triglyceride might be rapidly resecreted by the liver in newly assembled lipoproteins. Since VLDL production was markedly increased in the LPL0/A-I-LPL(L) mice, we favor this last option.

In adult LPL0/A-I-LPL(L) mice, adipose development occurred and the tissue attained relatively normal size. However, during the suckling period these mice gained less weight and had less body lipid mass. After switching to a high-carbohydrate chow diet, they gained weight and caught up to their littermates. A role for LPL in the development of adipose tissue and regulation of its size had been postulated more than $20 \mathrm{yr}$ ago (33). By producing mice selectively expressing LPL in specific tissues, LPL's actions and alternative pathways involved 
in adipocyte triglyceride accumulation have been defined. When LPL0 mice were crossed with animals expressing moderate amounts of LPL in muscle, the hypertriglyceridemia was corrected and the animals had normal life span (16). Adipose tissue development appeared normal; however, fatty acid analysis showed that glucose rather than circulating triglyceride fatty acids was used for fat storage in this tissue (21). Since triglyceride cannot be taken up by LPL-deficient tissues, adipose development in the LPL0/A-I-LPL(L) mice may require sufficient carbohydrate intake for conversion to fat stores. Presumably this did not occur during high fat suckling.

The mice expressing only liver LPL have hypertriglyceridemia, increased liver VLDL production, increased plasma ketones, and cachexia during high fat suckling. This metabolic pattern is similar to that seen during the host response to infection or cancer. Hypertriglyceridemia develops very fast after sepsis induction (8). This may result from activation of hormone-sensitive lipase leading to more liver fatty acid uptake, increased de novo fatty acid production, or the combination of decreased peripheral and increased liver LPL. Data documenting the time course of liver LPL expression in acute inflammatory conditions are not available. In chronic cachexia, however, a pattern of LPL expression similar to that in our model exists and could contribute to the abnormal lipid metabolism.

In summary, the mouse model created mimics lipid metabolism during both the neonatal period and, in part, cytokineinduced cachexia. We hypothesize that, like the LPL0/A-ILPL mice, in these metabolic situations fat-derived calories are shunted to the liver. This prevents disposal of energy for peripheral storage and oxidation. It also allows the generation of ketone bodies, which can be used as energy by peripheral tissues like brain and muscle instead of glucose. This may be required for survival during these metabolic situations because of insufficient carbohydrate availability. In addition, the triglyceride shift leads to the secretion of the newly acquired triglyceride, as was found in our liver-only LPL expressors as well as in TNF-induced cachectic mice. This futile cycle of LPL-mediated uptake and secretion of VLDL by the liver allows for greater ketone body production, and may have other beneficial effects during the host response to infection or cancer. During periods of normal food intake and metabolism, liver LPL may lead to metabolic inefficiency. Perhaps this is why it is suppressed in the normal adult animal.

\section{Acknowledgments}

The authors thank J.D. Smith and E. Sehayek for scientific discussions, R. Ramasamy, K. Aalto-Setälä, and Y.S. Kako for help with experiments, A. Strudl and A. Fuchsbichler for histological preparations, and I. Cho and L. Pang for their technical assistance.

This study was supported by National Institutes of Health grants HL-54591 (to J.L. Breslow) and HL-45095 (to I.J. Goldberg), and by the educational grant Me 1507/1-1 from the Deutsche Forschungsgemeinschaft (M. Merkel).

\section{References}

1. Olivecrona, G., and T. Olivecrona. 1995. Triglyceride lipases and atherosclerosis. Curr. Opin. Lipidol. 6:291-305.

2. Goldberg, I.J. 1996. Lipoprotein lipase and lipolysis: central roles in lipoprotein metabolism and atherogenesis. J. Lipid Res. 37:693-707.

3. Chajek, T., O. Stein, and Y. Stein. 1977. Pre- and post-natal development of lipoprotein lipase and hepatic triglyceride hydrolase activity in rat tissues. Atherosclerosis. 26:549-561.

4. Bensadoun, A., and T.L. Koh. 1977. Identification of an adipose tissuelike lipoprotein lipase in perfusates of chicken liver. J. Lipid Res. 18:768-773.

5. Semenkovich, C.F., S.H. Chen, M. Wims, C.C. Luo, W.H. Li, and L. Chan. 1989. Lipoprotein lipase and hepatic lipase mRNA tissue specific expression, developmental regulation, and evolution. J. Lipid Res. 30:423-431.

6. Yacoub, L.K., T.M. Vanni, and I.J. Goldberg. 1990. Lipoprotein lipase mRNA in neonatal and adult mouse tissues: comparison of normal and combined lipase deficiency (cld) mice assessed by in situ hybridization. J. Lipid Res. 31:1845-1852.

7. Tracey, K.J., and A. Cerami. 1992. Tumor necrosis factor in the malnutrition (cachexia) of infection and cancer. Am. J. Trop. Med. Hyg. 47:2-7.

8. Hardardottir, I., C. Grunfeld, and K.R. Feingold. 1994. Effects of endotoxin and cytokines on lipid metabolism. Curr. Opin. Lipidol. 5:207-215.

9. Enerback, S., H. Semb, J. Tavernier, G. Bjursell, and T. Olivecrona. 1988. Tissue-specific regulation of guinea pig lipoprotein lipase; effects of nutritional state and of tumor necrosis factor on mRNA levels in adipose tissue, heart and liver. Gene. 64:97-106.

10. Chajek-Shaul, T., G. Friedman, O. Stein, E. Shiloni, J. Etienne, and Y. Stein. 1989. Mechanism of the hypertriglyceridemia induced by tumor necrosis factor administration to rats. Biochim. Biophys. Acta. 1001:316-324.

11. Chajek-Shaul, T., E. Ziv, G. Friedman, J. Etienne, and J. Adler. 1988. Regulation of lipoprotein lipase activity in the sand rat: effect of nutritional state and cAMP modulation. Metabolism. 37:1152-1158.

12. Masuno, H., T. Tsujita, H. Nakanishi, A. Yoshida, R. Fukunishi, and H. Okuda. 1984. Lipoprotein lipase-like activity in the liver of mice with Sarcoma 180. J. Lipid Res. 25:419-427.

13. Walsh, A., Y. Ito, and J.L. Breslow. 1989. High levels of human apolipoprotein A-I in transgenic mice result in increased plasma levels of small high density lipoprotein (HDL) particles comparable to human HDL3. J. Biol. Chem. 264:6488-6494.

14. Levak-Frank, S., H. Radner, A. Walsh, R. Stollberger, G. Knipping, G. Hoefler, W. Sattler, P.H. Weinstock, J.L. Breslow, and R. Zechner. 1995. Muscle-specific overexpression of lipoprotein lipase causes a severe myopathy characterized by proliferation of mitochondria and peroxisomes in transgenic mice. J. Clin. Invest. 96:976-986.

15. Weinstock, P.H., C.L. Bisgaier, K. Aalto-Setala, H. Radner, R. Ramakrishnan, S. Levak-Frank, A.D. Essenburg, R. Zechner, and J.L. Breslow. 1995. Severe hypertriglyceridemia, reduced high density lipoprotein, and neonatal death in lipoprotein lipase knockout mice. J. Clin. Invest. 96:2555-2568.

16. Levak-Frank, S., P.H. Weinstock, T. Hayek, W. Hofmann, R. Verdery, J.L. Breslow, and R. Zechner. 1997. Induced mutant mice expressing lipoprotein lipase exclusively in muscle have subnormal triglycerides yet reduced HDL-cholesterol levels in plasma. J. Biol. Chem. 272:17182-17190.

17. Chomczynski, P., and N. Sacchi. 1987. Single-step method of RNA isolation by acid guanidinium thiocyanate-phenol-chloroform extraction. Anal. Biochem. 162:156-159.

18. Raisonnier, A., J. Etienne, F. Arnault, D. Brault, L. Noe, J.C. Chuat, and F. Galibert. 1995. Comparison of the cDNA and amino acid sequences of lipoprotein lipase in eight species. Comp. Biochem. Physiol. 111B:385-398.

19. Nilsson-Ehle, P., and M.C. Schotz. 1976. A stable, radioactive substrate emulsion for assay of lipoprotein lipase. J. Lipid Res. 17:536-541.

20. Goldberg, I.J., W.S. Blaner, T.M. Vanni, M. Moukides, and R. Ramakrishnan. 1990. Role of lipoprotein lipase in the regulation of high density lipoprotein apolipoprotein metabolism. Studies in normal and lipoprotein lipaseinhibited monkeys. J. Clin. Invest. 86:463-473.

21. Weinstock, P.H., S. Levak-Frank, L.C. Hudgins, H. Radner, J.M. Friedmann, R. Zechner, and J.L. Breslow. 1997. Lipoprotein lipase controls fatty acid entry into adipose tissue, but fat mass is preserved by endogenous synthesis in mice deficient in adipose tissue lipoprotein lipase. Proc. Natl. Acad. Sci. USA. 94:10261-10266.

22. Folch, J., M. Lees, and G.H. Sloane-Stanley. 1957. A simple method for the isolation and purification of total lipids from animal tissues. J. Biol. Chem 226:497-509.

23. Kates, M. 1972. Techniques in lipidology. In Laboratory Techniques in Biochemistry and Molecular Biology. Vol. 3. T.S. Work and E. Work, editors. Elsevier, New York. 267-610.

24. Plump, A.S., N. Azrolan, H. Odaka, L. Wu, X. Jiang, A. Tall, S. Eisenberg, and J.L. Breslow. 1997. ApoA-I knockout mice: characterization of HDL metabolism in homozygotes and identification of a post-RNA mechanism of apoA-I up-regulation in heterozygotes. J. Lipid Res. 38:1033-1047.

25. Havel, R.J., H.A. Eder, and J.H. Bragdon. 1955. The distribution and chemical composition of ultracentrifugally separated lipoproteins in human serum. J. Clin. Invest. 34:1345-1353.

26. Aalto-Setala, K., E.A. Fisher, X. Chen, T. Chajek-Shaul, T. Hayek, R. Zechner, A. Walsh, R. Ramakrishnan, H.N. Ginsberg, and J.L. Breslow. 1992. Mechanism of hypertriglyceridemia in human apolipoprotein (apo) CIII transgenic mice. Diminished very low density lipoprotein fractional catabolic rate associated with increased apo CIII and reduced apo E on the particles. J. Clin. Invest. 90:1889-1900.

27. Blanchette-Mackie, E.J., M.G. Wetzel, S.S. Chernick, J.R. Paterniti, Jr., 
W.V. Brown, and R.O. Scow. 1986. Effect of the combined lipase deficiency mutation (cld/cld) on ultrastructure of tissues in mice. Diaphragm, heart, brown adipose tissue, lung, and liver. Lab. Invest. 55:347-362.

28. Coleman, T., R.L. Seip, J.M. Gimble, D. Lee, N. Maeda, and C.F. Semenkovich. 1995. COOH-terminal disruption of lipoprotein lipase in mice is lethal in homozygotes, but heterozygotes have elevated triglycerides and impaired enzyme activity. J. Biol. Chem. 270:12518-12525.

29. Smart, J.L., D.N. Stephens, J. Tonkiss, N.S. Auestad, and J. Edmond. 1984. Growth and development of rats artificially reared on different milk-substitutes. Br. J. Nutr. 52:227-237.

30. Ensminger, A.H., M.E. Ensminger, E.K. James, and J.R.K. Robson.
1994. Encyclopedia of Nutrition. CRC Press, London. 1460 pp.

31. Hahn, P., and M. Novak. 1985. How important are carnitine and ketones for the newborn infant? FASEB (Fed. Am. Soc. Exp. Biol.) J. 44:23692373 .

32. Busch, S.J., R.L. Barnhart, G.A. Martin, M.C. Fitzgerald, M.T. Yates, S.J. Mao, C.E. Thomas, and R.L. Jackson. 1994. Human hepatic triglyceride lipase expression reduces high density lipoprotein and aortic cholesterol in cholesterol-fed transgenic mice. J. Biol. Chem. 269:16376-16382.

33. Greenwood, M.R.C. 1985. The relationship of enzyme activity to feeding behavior in rats: lipoprotein lipase as the metabolic gatekeeper. Int. J. Obes. 9:67-70. 